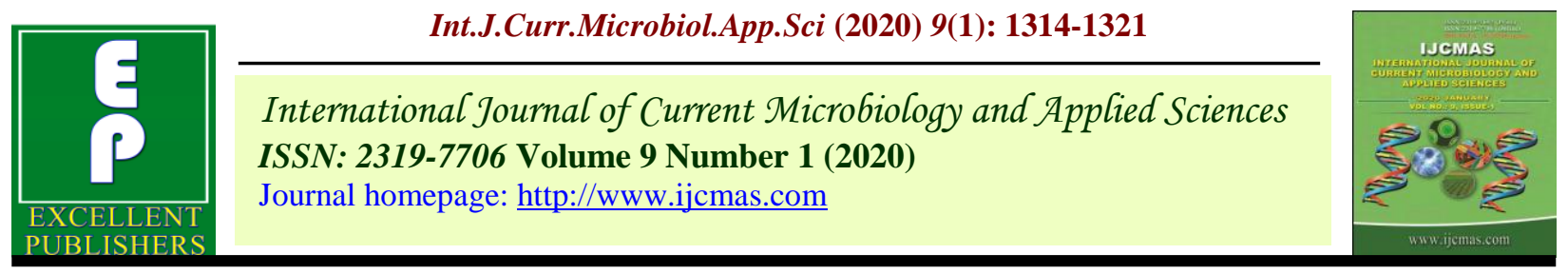

Original Research Article

https://doi.org/10.20546/ijcmas.2020.901.145

\title{
Effect of Drip Fertigation and Boron Foliar Spray on Growth, Seed Yield and Quality of Onion (Allium cepa l.) Cv. Palam lohit
}

\author{
Himangini* and H. S. Kanwar \\ Department of Seed Science and Technology, College of Horticulture, Dr. Y S Parmar \\ University of Horticulture and Forestry, Nauni, Solan-173230, India \\ *Corresponding author
}

\begin{tabular}{|l|}
\hline Ke y w o r d s \\
Onion, Drip \\
$\begin{array}{l}\text { Fertigation, Boron, } \\
\text { Plant Growth, Seed } \\
\text { Yield, Seed Quality }\end{array}$ \\
\hline Article Info \\
\hline $\begin{array}{l}\text { Accepted: } \\
\text { 15 December } 2019 \\
\text { Available Online: } \\
\text { 20 January } 2020\end{array}$ \\
\hline
\end{tabular}

Keywords

Onion, Drip

Fertigation, Boron,

Plant Growth, Seed

Yield, Seed Quality

Article Info

Accepted:

Available Online:

20 January 2020

\section{A B S T R A C T}

These investigations were conducted to study the influence of $\mathrm{N}$ and $\mathrm{K}$ drip fertigation along with boron foliar spray on growth, seed yield and seed quality of onion (Allium cepa L.). The experiments were carried out during two consecutive years (2014-15 and 2015-16) at experimental farm of Department of Seed Science and Technology, Dr. Y. S. Parmar University of Horticulture and Forestry, Nauni, Solan-173230 (H.P) using randomised block design. N and K fertilizers were applied through drip irrigation at three different levels viz; $100 \%, 80 \%$ and $60 \%$ of RDF as twenty different combinations in 10 equal splits at 15 days interval along with boron foliar spray. The results revealed that $\mathrm{N}$ and $\mathrm{K}$ fertigation along with boron foliar spray treatments gave significant results on plant growth, seed yield and quality parameters. Among all the treatments $\mathrm{N}_{100} \mathrm{~K}_{100} \mathrm{~B}_{1}(48.34 \mathrm{~cm})$ exhibited maximum plant height, minimum days to $50 \%$ flowering (151.00), number of flowering stalks per plant (7.73), number of umbels per plant (5.72), number of umblets per umbel (992.50), number of seeds/umbel (5143.27), seed yield /hectare (862.07), 1000 seed weight (4.39 g), germination \%age $(96.00 \%)$, seedling length $(15.18 \mathrm{~cm})$, seedling dry weight $(20.42 \mathrm{mg})$ and seedling vigour index I and II (1456.99 and 1961.01).

\section{Introduction}

Onion (Allium cepa L.) is a cool season vegetable crop and well adapted to Indian subcontinent. It is one of the most important vegetable cash crop used as green leaves as well as mature bulbs. India is the second largest producer of onion in the world with an area of 12.17 lakh ha and production of 192.99 lakh MT (Anonymous 2014). As per the estimates of National Seed Corporation, India needs about 4000 tonnes of certified seed of onion per year while the yearly production of seed has been estimated to about 6000 quintals (Singh, 2003), which indicates that there is an imperative need to produce more from less arable land and water. The continuous improvement in productivity with optimum 
utilization of water, fertilizer and natural recourses is essential for sustainability of any production system. Apart from the economic considerations, it is well established that the adverse effect of injudicious use of water and fertilizers can also have adverse implications on the environment.

Application of plant nutrients by dissolving them in irrigation water particularly with the drip system is termed as fertigation, offers a vast potential for more accurate and timely crop nutrition, preventing the leaching and voltalization losses of nutrients. The major advantages of fertigation are in saving of labour, appropriate timing of application of water and nutrients and their uniform distribution (Raina; 2002). Other advantages of fertigation lies in minimum leaching and voltalization losses, fertilizer use efficiency besides higher crop yields (Raina et al., 2011). In this method, liquid fertilizers as well as water soluble fertilizers are used and fertilizer use efficiency increased from $80-90 \%$. Apart from major nutrients, micronutrients also play an important role in seed production. Foliar spraying of these nutrients may help the plant to uptake nutrients better than soil application. Generally, the plant requires a wide variety of elements to improve the growth and yield. Among these, boron is an essential element required for the development of cell wall, cell differentiation, root elongation and shoot growth. It has been involved in carbohydrate synthesis, uptake of $\mathrm{Ca}^{2+}$ and absorption of $\mathrm{NO}^{3}$. Boron is essential not only for the formation of the pollen and ovary, but also for the subsequent development of the seed. Thus, good quality seed is also one of the important means to increase productivity in any seed crop. In this view, the present investigations were conducted to know the effect of drip fertigation and boron foliar spray on growth, seed yield, quality of onion (Allium cepa L.) in cv. Palam Lohit.

\section{Materials and Methods}

These investigations were carried out for two consecutive years (2014-15 and 2015-16) at the experimental farm of the Department of Seed Science and Technology, Dr. Y S Parmar University of Horticulture and Forestry, Nauni, Solan (latitude of $35.5^{\circ} \mathrm{N}$, longitude of $77.8^{\circ} \mathrm{E}$ and altitude of $1250 \mathrm{amsl}$ ). The soils of area belong to Typic Eutrochrept at sub group level according to Soil Taxonomy of USDA. Salient physio- chemical properties of the experimental soil for $0-20 \mathrm{~cm}$ depth are presented in Table 1. Climate of the area is generally sub temperate and semi humid characterized by cold winters. Annual rainfall 0.00 to $213.6 \mathrm{~mm}$, mean temperature ranges between $9.9^{\circ} \mathrm{C}-23.7^{\circ} \mathrm{C}$, relative humidity 45 $63 \%$. The soil was prepared by one round of ploughing and harrowing. Disease free, healthy, uniform sized bulbs of about 60-65 g in weight were selected and were planted in furrows covered with soil. Each experimental unit consisted of a bed of $1.5 \times 1.2 \mathrm{~m}$, containing two rows of plants spaced $45 \times 30$ $\mathrm{cm}$. The drip fertigation and foliar spray treatments details are given below:

$\mathbf{N}_{\text {RDF }} \mathbf{K}_{\text {RDF }} \mathbf{B}_{\mathbf{0}}$ : $\quad$ Conventional

application of Nitrogen and Potassium fertilizers + distilled water spray

$\mathbf{N}_{\text {RDF }} \mathbf{K}_{\text {RDF }} \mathbf{B}_{1}$ : $\quad$ Conventional

application of Nitrogen and Potassium fertilizers + distilled water spray

$\mathbf{N}_{100} \mathbf{K}_{100} \mathbf{B}_{\mathbf{0}} \quad$ : $\quad$ Fertigation of $100 \%$ of nitrogen and $100 \%$ of potassium + distilled water spray

$\mathbf{N}_{100} \mathbf{K}_{100} \mathbf{B}_{1}$ : $\quad$ Fertigation of $100 \%$ of nitrogen and $100 \%$ of potassium + boron foliar spray

$\mathbf{N}_{\mathbf{1 0 0}} \mathbf{K}_{\mathbf{8 0}} \mathbf{B}_{\mathbf{0}}$ : $\quad$ Fertigation of $100 \%$ of nitrogen and $80 \%$ of potassium + distilled water spray $\mathbf{N}_{\mathbf{1 0 0}} \mathbf{K}_{\mathbf{8 0}} \mathbf{B}_{\mathbf{1}} \quad$ : $\quad$ Fertigation of $100 \%$ of nitrogen and $80 \%$ of potassium + boron foliar spray

$\mathbf{N}_{100} K_{60} B_{0} \quad$ : $\quad$ Fertigation of $100 \%$ of 
nitrogen and $60 \%$ of potassium + distilled water spray

$\mathbf{N}_{\mathbf{1 0 0}} \mathbf{K}_{\mathbf{6 0}} \mathbf{B}_{\mathbf{1}} \quad$ : $\quad$ Fertigation of $100 \%$ of nitrogen and $60 \%$ of potassium + boron foliar spray

$\mathbf{N}_{\mathbf{8 0}} \mathbf{K}_{\mathbf{1 0 0}} \mathbf{B}_{\mathbf{0}} \quad$ : $\quad$ Fertigation of $80 \%$ of nitrogen and $100 \%$ of potassium + distilled water spray

$\mathbf{N}_{\mathbf{8 0}} \mathbf{K}_{100} \mathbf{B}_{1} \quad$ : $\quad$ Fertigation of $80 \%$ of nitrogen and $100 \%$ of potassium + boron foliar spray

$\mathbf{N}_{\mathbf{8 0}} \mathbf{K}_{\mathbf{8 0}} \mathbf{B}_{\mathbf{0}} \quad$ : $\quad$ Fertigation of $80 \%$ of nitrogen and $80 \%$ of potassium + distilled water spray

$\mathbf{N}_{\mathbf{8 0}} \mathbf{K}_{\mathbf{8 0}} \mathbf{B}_{\mathbf{1}} \quad$ : $\quad$ Fertigation of $80 \%$ of nitrogen and $80 \%$ of potassium + boron foliar spray

$\mathbf{N}_{\mathbf{8 0}} \mathbf{K}_{\mathbf{6 0}} \mathbf{B}_{\mathbf{0}} \quad$ : $\quad$ Fertigation of $80 \%$ of nitrogen and $60 \%$ of potassium + distilled water spray

$\mathbf{N}_{\mathbf{8 0}} \mathbf{K}_{60} \mathbf{B}_{1}$ : $\quad$ Fertigation of $80 \%$ of nitrogen and $60 \%$ of potassium + boron foliar spray

$\mathbf{N}_{\mathbf{6 0}} \mathbf{K}_{\mathbf{1 0 0}} \mathbf{B}_{\mathbf{0}} \quad$ : $\quad$ Fertigation of $60 \%$ of nitrogen and $100 \%$ of potassium + distilled water spray

$\mathbf{N}_{60} \mathbf{K}_{100} \mathbf{B}_{1}$ : $\quad$ Fertigation of $60 \%$ of nitrogen and $100 \%$ of potassium + boron foliar spray

$\mathbf{N}_{60} \mathbf{K}_{80} \mathbf{B}_{\mathbf{0}} \quad$ : $\quad$ Fertigation of $60 \%$ of nitrogen and $80 \%$ of potassium + distilled water spray

$\mathbf{N}_{60} \mathbf{K}_{\mathbf{8 0}} \mathbf{B}_{1} \quad$ : $\quad$ Fertigation of $60 \%$ of nitrogen and $80 \%$ of potassium + boron foliar spray

$\mathbf{N}_{60} \mathbf{K}_{60} \mathbf{B}_{\mathbf{0}} \quad$ : $\quad$ Fertigation of $60 \%$ of nitrogen and $60 \%$ of potassium + distilled water spray

$\mathbf{N}_{60} \mathbf{K}_{60} \mathbf{B}_{1} \quad$ : $\quad$ Fertigation of $60 \%$ of nitrogen and $60 \%$ of potassium + boron foliar spray

\section{Results and Discussion}

The analysis of variance indicated significant differences for the effect of different levels of fertigation on various plant attributed characters whereas; boron spray shows significant differences for the seed yield and quality parameters as the treatment was applied before flowering stage. Thus, the results obtained from the present investigation have been discussed in the following sub heads. Significant differences for the effect of different levels of fertigation on various plant and flowering attributed characters of onion whereas, boron foliar spray shows nonsignificant differences (Table 1). Among all the treatment combinations $\mathrm{N}_{100} \mathrm{~K}_{100} \mathrm{~B}_{1}$ gave maximum plant height $(48.34 \mathrm{~cm})$, flowering stalks per plant (7.73), number of umbels per plant (5.72), umblets per umbel (992.50), seeds per plant (5143.27), yield per hectare $(862.07 \mathrm{Kg})$ and minimum days taken for 50 $\%$ flowering (151.00 days) which was also statistically at par with treatment $\mathrm{N}_{100} \mathrm{~K}_{80} \mathrm{~B}_{1}$. The results may be attributed to the fact that nitrogen is an essential part of chlorophyll and nucleic acids, which played a major role in promoting plant growth on the other hand potassium plays an important role in translocation of photosynthesis thus the higher concentration of both nitrogen and potassium has the ability to increase the number of cells of leaves, cell size and overall vegetative and reproductive growth of the plants. The results of the present investigation in terms of plant height are in accordance with the findings reported earlier by Damaranay et al., (2016) in onion, Rahim et al., (1997) in onion, Kabir et al., (2013), Marks et al., (2006) in carrot and Jadhao et al., (1999) in radish.

The increased seed recovery $\%$ might be due to the effect of micronutrients which play a decisive role in improving the productivity of the crop. In fact, boron helps in arresting flower drop and improves the seed setting which concerned with sugar translocation from complex compounds like carbohydrates and translocated them at greater ease. 
Table.1 Effect of different levels of fertigation and boron foliar spray on plant and seed yield attributes in onion

\begin{tabular}{|c|c|c|c|c|c|c|c|c|c|c|c|c|c|c|c|c|c|c|c|c|c|}
\hline \multirow[t]{3}{*}{ Treatments } & \multicolumn{3}{|c|}{$\begin{array}{l}\text { Plant height } \\
\text { (cm) }\end{array}$} & \multicolumn{3}{|c|}{$\begin{array}{l}\text { Days to } 50 \% \\
\text { flowering }\end{array}$} & \multicolumn{3}{|c|}{$\begin{array}{c}\text { Number of } \\
\text { flowering stalks } \\
\text { per plant }\end{array}$} & \multicolumn{3}{|c|}{$\begin{array}{l}\text { Number of umbels } \\
\text { per plant }\end{array}$} & \multicolumn{3}{|c|}{$\begin{array}{c}\text { Number of umblets } \\
\text { per umbel }\end{array}$} & \multicolumn{3}{|c|}{$\begin{array}{l}\text { Number of } \\
\text { seeds/umbel }\end{array}$} & \multicolumn{3}{|c|}{$\begin{array}{l}\text { Seed yield /ha } \\
(\mathrm{kg})\end{array}$} \\
\hline & \multicolumn{21}{|c|}{ Boron treatment } \\
\hline & $\mathbf{B}_{0}$ & $\mathbf{B}_{1}$ & $\begin{array}{c}\text { Mea } \\
\mathbf{n}\end{array}$ & $\mathbf{B}_{0}$ & $\mathbf{B}_{1}$ & $\begin{array}{c}\text { Mea } \\
\mathbf{n}\end{array}$ & $\mathbf{B}_{0}$ & $\mathbf{B}_{1}$ & $\begin{array}{c}\text { Mea } \\
\mathbf{n}\end{array}$ & $\mathbf{B}_{0}$ & $\mathbf{B}_{1}$ & $\begin{array}{l}\text { Me } \\
\text { an }\end{array}$ & $\mathbf{B}_{0}$ & $\mathbf{B}_{1}$ & $\begin{array}{c}\text { Mea } \\
\text { n }\end{array}$ & $\mathbf{B}_{0}$ & $\mathbf{B}_{1}$ & Mean & $\mathbf{B}_{0}$ & $\mathbf{B}_{1}$ & Mean \\
\hline $\begin{array}{l}\mathbf{N}_{\text {RDF }} \mathbf{K}_{\mathbf{R}} \\
\text { DF }\end{array}$ & $\begin{array}{c}45.6 \\
2\end{array}$ & $\begin{array}{c}46.2 \\
8\end{array}$ & $\begin{array}{c}45.9 \\
5\end{array}$ & $\begin{array}{c}149 . \\
00\end{array}$ & $\begin{array}{c}149 . \\
33\end{array}$ & $\begin{array}{c}149 . \\
17\end{array}$ & 7.32 & 7.28 & 7.30 & 5.45 & 5.37 & 5.41 & $\begin{array}{c}970.1 \\
7\end{array}$ & $\begin{array}{c}969 . \\
83\end{array}$ & $\begin{array}{c}970 . \\
00\end{array}$ & $\begin{array}{c}4281 . \\
22\end{array}$ & $\begin{array}{c}4751 . \\
52\end{array}$ & $\begin{array}{c}4516 . \\
37\end{array}$ & $\begin{array}{c}831 . \\
56\end{array}$ & $\begin{array}{c}848 . \\
59\end{array}$ & 840.08 \\
\hline $\mathbf{N}_{100} K_{100}$ & $\begin{array}{c}48.3 \\
4\end{array}$ & $\begin{array}{c}48.6 \\
7\end{array}$ & $\begin{array}{c}48.5 \\
0\end{array}$ & $\begin{array}{c}150 . \\
67\end{array}$ & $\begin{array}{c}151 . \\
00\end{array}$ & $\begin{array}{c}150 . \\
83\end{array}$ & 7.73 & 7.70 & 7.72 & 5.72 & 5.65 & 5.68 & $\begin{array}{c}992.5 \\
0\end{array}$ & $\begin{array}{c}992 . \\
00\end{array}$ & $\begin{array}{c}992 . \\
25\end{array}$ & $\begin{array}{c}5143 . \\
27\end{array}$ & $\begin{array}{c}5744 . \\
67\end{array}$ & $\begin{array}{c}5443 . \\
97\end{array}$ & $\begin{array}{c}862 . \\
07\end{array}$ & $\begin{array}{c}886 . \\
96\end{array}$ & 874.52 \\
\hline $\mathbf{N}_{100} K_{80}$ & $\begin{array}{c}46.7 \\
5\end{array}$ & $\begin{array}{c}47.1 \\
7\end{array}$ & $\begin{array}{c}46.9 \\
6\end{array}$ & $\begin{array}{c}151 . \\
00\end{array}$ & $\begin{array}{c}150 . \\
67\end{array}$ & $\begin{array}{c}150 . \\
83\end{array}$ & 7.43 & 7.44 & 7.44 & 5.60 & 5.45 & 5.53 & $\begin{array}{c}977.0 \\
0\end{array}$ & $\begin{array}{c}975 . \\
83\end{array}$ & $\begin{array}{c}976 . \\
42\end{array}$ & $\begin{array}{c}4770 . \\
43\end{array}$ & $\begin{array}{c}5361 . \\
07\end{array}$ & $\begin{array}{c}5065 . \\
75\end{array}$ & $\begin{array}{c}845 . \\
33\end{array}$ & $\begin{array}{c}866 . \\
07\end{array}$ & 855.70 \\
\hline $\mathbf{N}_{100} K_{60}$ & $\begin{array}{c}45.1 \\
8\end{array}$ & $\begin{array}{c}45.7 \\
5\end{array}$ & $\begin{array}{c}45.4 \\
6\end{array}$ & $\begin{array}{c}150 . \\
00\end{array}$ & $\begin{array}{c}150 . \\
33\end{array}$ & $\begin{array}{c}150 . \\
17\end{array}$ & 7.15 & 7.21 & 7.18 & 5.47 & 5.35 & 5.41 & $\begin{array}{c}967.3 \\
3\end{array}$ & $\begin{array}{c}966 . \\
67\end{array}$ & $\begin{array}{c}967 . \\
00\end{array}$ & $\begin{array}{c}4010 . \\
41\end{array}$ & $\begin{array}{c}4408 . \\
04\end{array}$ & $\begin{array}{c}4209 \\
23\end{array}$ & $\begin{array}{c}812 . \\
59\end{array}$ & $\begin{array}{c}832 . \\
74\end{array}$ & 822.67 \\
\hline $\mathbf{N}_{80} K_{100}$ & $\begin{array}{c}44.0 \\
0\end{array}$ & $\begin{array}{c}44.5 \\
1\end{array}$ & $\begin{array}{c}44.2 \\
5\end{array}$ & $\begin{array}{c}148 . \\
33\end{array}$ & $\begin{array}{c}149 . \\
00\end{array}$ & $\begin{array}{c}148 . \\
67\end{array}$ & 6.82 & 6.92 & 6.87 & 5.38 & 5.18 & 5.28 & $\begin{array}{c}963.0 \\
0\end{array}$ & $\begin{array}{c}962 . \\
50\end{array}$ & $\begin{array}{c}962 . \\
75\end{array}$ & $\begin{array}{c}3172 . \\
98\end{array}$ & $\begin{array}{c}3949 . \\
34\end{array}$ & $\begin{array}{c}3561 \\
16\end{array}$ & $\begin{array}{c}792 . \\
59\end{array}$ & $\begin{array}{c}807 . \\
41\end{array}$ & 800.00 \\
\hline $\mathbf{N}_{80} K_{80}$ & $\begin{array}{c}42.4 \\
1\end{array}$ & $\begin{array}{c}42.9 \\
8\end{array}$ & $\begin{array}{c}42.6 \\
9\end{array}$ & $\begin{array}{c}147 . \\
67\end{array}$ & $\begin{array}{c}147 . \\
33\end{array}$ & $\begin{array}{c}147 . \\
50\end{array}$ & 6.40 & 6.47 & 6.43 & 5.22 & 5.00 & 5.11 & $\begin{array}{c}917.3 \\
3\end{array}$ & $\begin{array}{c}916 . \\
33\end{array}$ & $\begin{array}{c}916 . \\
83\end{array}$ & $\begin{array}{c}2440 . \\
51\end{array}$ & $\begin{array}{c}3264 . \\
71\end{array}$ & $\begin{array}{c}2852 . \\
61\end{array}$ & $\begin{array}{c}739 . \\
11\end{array}$ & $\begin{array}{c}773 . \\
19\end{array}$ & 756.15 \\
\hline $\mathbf{N}_{80} K_{60}$ & $\begin{array}{c}41.5 \\
0\end{array}$ & $\begin{array}{c}41.9 \\
5\end{array}$ & $\begin{array}{c}41.7 \\
2\end{array}$ & $\begin{array}{c}148 . \\
00\end{array}$ & $\begin{array}{c}147 . \\
67\end{array}$ & $\begin{array}{c}147 . \\
83\end{array}$ & 6.42 & 6.48 & 6.45 & 5.07 & 4.82 & 4.94 & $\begin{array}{c}862.6 \\
7\end{array}$ & $\begin{array}{c}861 . \\
17\end{array}$ & $\begin{array}{c}861 . \\
92\end{array}$ & $\begin{array}{c}1564 . \\
05\end{array}$ & $\begin{array}{c}1983 . \\
34\end{array}$ & $\begin{array}{c}1773 . \\
70\end{array}$ & $\begin{array}{c}685 . \\
93\end{array}$ & $\begin{array}{c}719 . \\
11\end{array}$ & 702.52 \\
\hline $\mathbf{N}_{60} K_{100}$ & $\begin{array}{c}43.2 \\
5\end{array}$ & $\begin{array}{c}43.7 \\
7\end{array}$ & $\begin{array}{c}43.5 \\
1\end{array}$ & $\begin{array}{c}147 . \\
00\end{array}$ & $\begin{array}{c}146 . \\
33\end{array}$ & $\begin{array}{c}146 . \\
67\end{array}$ & 6.18 & 6.13 & 6.16 & 4.85 & 4.67 & 4.76 & $\begin{array}{c}945.8 \\
3\end{array}$ & $\begin{array}{c}945 . \\
00\end{array}$ & $\begin{array}{c}945 . \\
42\end{array}$ & $\begin{array}{c}2939 . \\
02\end{array}$ & $\begin{array}{c}3393 . \\
37\end{array}$ & $\begin{array}{c}3166 . \\
20\end{array}$ & $\begin{array}{c}732 . \\
59\end{array}$ & $\begin{array}{c}782 . \\
07\end{array}$ & 757.33 \\
\hline $\mathbf{N}_{60} K_{80}$ & $\begin{array}{c}42.1 \\
5\end{array}$ & $\begin{array}{c}42.6 \\
6\end{array}$ & $\begin{array}{c}42.4 \\
1\end{array}$ & $\begin{array}{c}146 . \\
00\end{array}$ & $\begin{array}{c}146 . \\
67\end{array}$ & $\begin{array}{c}146 . \\
33\end{array}$ & 5.95 & 5.92 & 5.93 & 4.42 & 4.32 & 4.37 & $\begin{array}{c}891.1 \\
7\end{array}$ & $\begin{array}{c}890 . \\
33\end{array}$ & $\begin{array}{c}890 . \\
75\end{array}$ & $\begin{array}{c}2224 . \\
9\end{array}$ & $\begin{array}{c}2726 . \\
25\end{array}$ & $\begin{array}{c}2475 . \\
56\end{array}$ & $\begin{array}{c}680 . \\
59\end{array}$ & $\begin{array}{c}734 . \\
22\end{array}$ & 707.41 \\
\hline $\mathbf{N}_{60} K_{60}$ & $\begin{array}{c}40.1 \\
5\end{array}$ & $\begin{array}{c}40.6 \\
4\end{array}$ & $\begin{array}{c}40.3 \\
9\end{array}$ & $\begin{array}{c}145 . \\
33\end{array}$ & $\begin{array}{c}145 . \\
67\end{array}$ & $\begin{array}{c}145 . \\
50\end{array}$ & 5.73 & 5.73 & 5.73 & 4.53 & 4.23 & 4.38 & $\begin{array}{c}854.0 \\
0\end{array}$ & $\begin{array}{c}853 . \\
17\end{array}$ & $\begin{array}{c}853 . \\
58\end{array}$ & $\begin{array}{c}1111 . \\
99\end{array}$ & $\begin{array}{c}1846 . \\
96\end{array}$ & $\begin{array}{c}1479 \\
48\end{array}$ & $\begin{array}{c}620 . \\
30\end{array}$ & $\begin{array}{c}694 . \\
96\end{array}$ & 657.63 \\
\hline Mean & $\begin{array}{c}43.9 \\
3\end{array}$ & $\begin{array}{c}44.4 \\
4\end{array}$ & & $\begin{array}{c}148 . \\
30\end{array}$ & $\begin{array}{c}148 . \\
40\end{array}$ & & 6.71 & 6.73 & & 5.17 & 5.00 & & $\begin{array}{c}934.1 \\
0\end{array}$ & $\begin{array}{c}933 . \\
28\end{array}$ & & $\begin{array}{c}3165 . \\
88\end{array}$ & $\begin{array}{c}3742 . \\
93\end{array}$ & & $\begin{array}{c}760 . \\
27\end{array}$ & $\begin{array}{c}794 . \\
53\end{array}$ & \\
\hline & & & & & & & & & & & .05) & & & & & & & & & & \\
\hline $\mathbf{T}$ & & & 1.60 & & & 0.85 & & & 0.40 & & & 0.34 & & & $\begin{array}{c}16.0 \\
0\end{array}$ & & & 59.31 & & & 6.70 \\
\hline B & & & NS & & & NS & & & NS & & & NS & & & NS & & & $\begin{array}{c}132.6 \\
2\end{array}$ & & & 14.99 \\
\hline $\mathbf{T} \mathbf{X} \mathbf{B}$ & & & NS & & & NS & & & NS & & & NS & & & NS & & & $\begin{array}{c}187.5 \\
6\end{array}$ & & & 21.19 \\
\hline
\end{tabular}


Table.2 Effect of different levels of fertigation and boron spray on quality attributes of harvested onion seed

\begin{tabular}{|c|c|c|c|c|c|c|c|c|c|c|c|c|c|c|c|c|c|c|}
\hline \multirow[t]{3}{*}{ Treatments } & \multicolumn{3}{|c|}{$\begin{array}{l}1000 \text { seed weight } \\
\text { (g) }\end{array}$} & \multicolumn{3}{|c|}{ Germination (\%) } & \multicolumn{3}{|c|}{ Seedling length (cm) } & \multicolumn{3}{|c|}{$\begin{array}{c}\text { Seedling dry weight } \\
\text { (mg) }\end{array}$} & \multicolumn{3}{|c|}{ Seedling vigour I } & \multicolumn{3}{|c|}{ Seedling vigour II } \\
\hline & \multicolumn{18}{|c|}{ Boron Treatment } \\
\hline & $\mathbf{B}_{0}$ & $\mathbf{B}_{1}$ & Mea & $\mathbf{B}_{0}$ & $\mathbf{B}_{1}$ & Mean & $\mathbf{B}_{0}$ & $\mathbf{B}_{1}$ & Mean & $\mathbf{B}_{0}$ & $\mathbf{B}_{1}$ & Mean & $\mathbf{B}_{0}$ & $\mathbf{B}_{1}$ & Mean & $\mathbf{B}_{0}$ & $\mathbf{B}_{1}$ & Mean \\
\hline $\mathbf{N}_{\mathrm{RDF}} \mathrm{K}_{\mathrm{RDF}}$ & 3.24 & 4.26 & 3.75 & $\begin{array}{c}91.50 \\
(72.53)\end{array}$ & $\begin{array}{c}93.92 \\
(74.85)\end{array}$ & $\begin{array}{c}92.71 \\
(73.68)\end{array}$ & 14.86 & 15.11 & 14.99 & 20.27 & 20.34 & 20.30 & 1359.98 & 1419.36 & 1389.67 & 1855.33 & 1910.44 & 1882.89 \\
\hline $\mathbf{N}_{100} \mathbf{K}_{100}$ & 3.71 & 4.39 & 4.05 & $\begin{array}{c}94.83 \\
(76.63)\end{array}$ & $\begin{array}{c}96.00 \\
(77.09)\end{array}$ & $\begin{array}{c}95.42 \\
(76.86)\end{array}$ & 15.01 & 15.18 & 15.10 & 20.30 & 20.42 & 20.36 & 1423.55 & 1456.99 & 1440.27 & 1924.98 & 1961.01 & 1943.00 \\
\hline $\mathbf{N}_{100} K_{80}$ & 3.32 & 4.34 & 3.83 & $\begin{array}{c}92.33 \\
(73.90)\end{array}$ & $\begin{array}{c}94.63 \\
(75.02)\end{array}$ & $\begin{array}{c}93.48 \\
(74.46)\end{array}$ & 14.95 & 15.17 & 15.06 & 20.22 & 20.27 & 20.24 & 1380.07 & 1436.83 & 1408.45 & 1867.14 & 1919.06 & 1893.10 \\
\hline $\mathbf{N}_{100} \mathbf{K}_{60}$ & 3.09 & 4.21 & 3.65 & $\begin{array}{c}90.83 \\
(72.19)\end{array}$ & $\begin{array}{c}92.67 \\
(73.20)\end{array}$ & $\begin{array}{c}91.75 \\
(72.69)\end{array}$ & 14.73 & 15.03 & 14.88 & 20.04 & 20.09 & 20.07 & 1337.93 & 1393.23 & 1365.58 & 1820.35 & 1863.16 & 1841.75 \\
\hline $\mathbf{N}_{80} \mathbf{K}_{100}$ & 2.99 & 4.07 & 3.53 & $\begin{array}{c}89.00 \\
(69.76)\end{array}$ & $\begin{array}{c}90.42 \\
(71.38)\end{array}$ & $\begin{array}{c}89.71 \\
(70.55)\end{array}$ & 14.48 & 14.85 & 14.67 & 19.81 & 19.99 & 19.90 & 1289.37 & 1343.07 & 1316.22 & 1764.46 & 1807.98 & 1786.22 \\
\hline $\mathbf{N}_{80} \mathbf{K}_{80}$ & 2.92 & 3.85 & 3.38 & $\begin{array}{c}85.50 \\
(67.19)\end{array}$ & $\begin{array}{c}89.17 \\
(70.02)\end{array}$ & $\begin{array}{c}87.33 \\
(68.61)\end{array}$ & 13.99 & 14.50 & 14.25 & 19.52 & 19.69 & 19.60 & 1196.78 & 1293.81 & 1245.30 & 1669.30 & 1756.40 & 1712.85 \\
\hline $\mathbf{N}_{80} K_{60}$ & 2.81 & 3.63 & 3.22 & $\begin{array}{c}82.33 \\
(66.14)\end{array}$ & $\begin{array}{c}86.92 \\
(68.14)\end{array}$ & $\begin{array}{c}84.63 \\
(67.14)\end{array}$ & 13.50 & 14.15 & 13.83 & 19.20 & 19.38 & 19.29 & 1110.16 & 1230.17 & 1170.16 & 1578.60 & 1685.42 & 1632.01 \\
\hline $\mathbf{N}_{60} K_{100}$ & 3.07 & 3.92 & 3.49 & $\begin{array}{c}85.25 \\
(65.25)\end{array}$ & $\begin{array}{c}84.00 \\
(65.25)\end{array}$ & $\begin{array}{c}84.63 \\
(65.25)\end{array}$ & 14.22 & 14.66 & 14.44 & 19.67 & 19.82 & 19.74 & 1213.97 & 1232.39 & 1223.18 & 1680.10 & 1666.65 & 1673.38 \\
\hline $\mathbf{N}_{60} K_{80}$ & 2.86 & 3.75 & 3.30 & $\begin{array}{c}82.58 \\
(64.26)\end{array}$ & $\begin{array}{c}83.00 \\
(64.63)\end{array}$ & $\begin{array}{l}82.79 \\
64.44)\end{array}$ & 13.73 & 14.31 & 14.02 & 19.36 & 19.50 & 19.43 & 1134.63 & 1188.43 & 1161.53 & 1600.96 & 1619.86 & 1610.41 \\
\hline $\mathbf{N}_{60} K_{60}$ & 2.78 & 3.55 & 3.16 & $\begin{array}{c}80.58 \\
(63.41)\end{array}$ & $\begin{array}{c}80.42 \\
(63.17)\end{array}$ & $\begin{array}{c}80.50 \\
(63.29)\end{array}$ & 13.23 & 13.95 & 13.59 & 19.02 & 19.21 & 19.12 & 1066.49 & 1122.28 & 1094.39 & 1533.77 & 1545.66 & 1539.71 \\
\hline Mean & 3.08 & 4.00 & 3.54 & $\begin{array}{c}87.48 \\
(69.12)\end{array}$ & $\begin{array}{c}89.11 \\
(74.83)\end{array}$ & $\begin{array}{c}88.29 \\
(69.70)\end{array}$ & 14.27 & 14.69 & 14.48 & 19.74 & 19.87 & 19.80 & 1251.29 & 1311.65 & 1281.47 & 1729.50 & 1773.57 & 1751.53 \\
\hline & & & & & & & & & CD ( & 05) & & & & & & & & \\
\hline $\mathbf{T}$ & & & & 0.06 & & & 0.037 & & & 0.083 & & & 6.100 & & & & & \\
\hline B & & & & 0.03 & & & 0.117 & & & 0.011 & & & 13.640 & & & 13. & & \\
\hline T X B & & & & 0.08 & & & 0.026 & & & 0.036 & & & 19.290 & & & & 92 & \\
\hline
\end{tabular}


Chemical characteristics of the soil to a depth $0-20 \mathrm{~cm}$, sampled prior to the experiment

\begin{tabular}{|l|c|}
\hline \multicolumn{1}{|c|}{ Properties } & Initial values \\
\hline pH $(\mathbf{1 : 2})$ & 6.21 \\
\hline $\mathbf{E C}\left(\mathbf{d S m}^{-\mathbf{1}}\right)$ & 0.25 \\
\hline Organic carbon $\left(\mathrm{g} \mathrm{kg}^{-\mathbf{1}}\right)$ & 8.10 \\
\hline Available $\mathbf{N}\left(\mathrm{kg} \mathrm{ha}^{-1}\right)$ & 258.0 \\
\hline Available P $\left(\mathrm{kg} \mathrm{ha}^{-\mathbf{1}}\right)$ & 53.70 \\
\hline Available K $\left(\mathrm{kg} \mathrm{ha}^{-1}\right)$ & 112.8 \\
\hline
\end{tabular}

Boron plays a greater role in nitrogen based synthesis or utilization and involved in RNA metabolism (Deepika and Anita, 2015) similar results were reported by Ali et al., (2007) in onion, Hamsaveni et al., (2003) in tomato. The delayed flowering by the plants fertilized with $100 \%$ nitrogen might have been due to the influence of higher level of nitrogen in delaying initiation of flowering caused by prolonged vegetative phase (Rajangam, 1991). Similar results were found by He and Chen (1996) in tomato and Suthar et al., (2005) in eggplant.

An analysis of data presented in Table 2 significantly indicated that $\mathrm{N}_{100} \mathrm{~K}_{100} \mathrm{~B}_{1}$ reported maximum 1000 seed weight $(4.39 \mathrm{~g})$, germination \% (96.00\%), seedling length $(15.18 \mathrm{~cm})$, seedling dry weight $(20.42 \mathrm{mg})$ and Seedling vigour index I and II (1456.99 and 1961.01) respectively, which was at par with $\mathrm{N}_{100} \mathrm{~K}_{80} \mathrm{~B}_{1}$. The results were in consonance with Singh et al., 1994 who reported that higher germination \%age in seeds is due to better mineral utilization of plants treated with drip fertigation accompanied with enhancement of photosynthesis and greater diversion of food material to seeds. The higher seed quality attributes correlating with higher level of water soluble fertilizers could be attributed to translocation of more carbohydrates due to high nitrogen levels which in turn increases the reserve food material in seed and increase 1000 seed weight. Such beneficial results are also reported with foliar application of boron which is involved in development of cell wall, cell differentiation, root and shoot elongation. It is also involved in ovary developments, seed development and maturity (Sharma; 1995, Verma et al., 1995). The results are in conformity with Chavan (1998) and Darwati et al., (1990) in sesame. This study supported the possibility that potassium plays an important role in this translocation of metabolites for the development of seed. The improvement in root and shoot length of seedling due to boron ascribed to the efficient protein synthesis and better source to sink relationship which resulted in better development of seeds giving rise to higher germination and vigour. These results were in collaborative with Dileepkumar et al., (2009) in cowpea, Arvind Kumar et al., (2012) in bitter gourd.

Thus it is concluded that $\mathrm{N}_{100} \mathrm{~K}_{100}$ doses of fertilizer applied through drip irrigation and boron@ @ $0.01 \%$ foliar spray (before flowering stage) treatment is effective in increasing the plant growth, seed yield and quality of onion as compared to conventional method of applying nutrients to soil.

\section{Acknowledgements}

The authors are greatly thankful to the Head and Professor of Department of Vegetable Science and Seed Science and Technology, Dr. Yashwant Singh Parmar University of Horticulture and Forestry, Nauni, Solan for providing official support during the research. 


\section{References}

Ali, M. K., Alam, M. F., Alam, M. N., Islam, M. S. and Khandaker, S. M. A. T. 2007. Effect of Nitrogen and Potassium Level on Yield and Quality Seed Production of Onion. Journal of Applied Sciences Research. 3(12): 1889-1899.

Anonymous, 2014.www.NHCB.com

Arvindkumar, P. R., Vasudevan, S. N., Patil, M. G. and Rajarajeshwari, C. 2012. Influence of NAA, triacontanol and boron spray on seed yield and quality of bitter gourd (Momordica charantia) cv. Pusa Visesh. The Asian Journal of Horticulture. 7(1):36-39.

Chavan, K. K. 1998. Influence of Seed Size and Mother Plant Nutrition on Seed Yield and quality in Sesamum (Sesamum indicum L.) M.Sc. Thesis, University of Agricultural Sciences, Dharwad.

Darwati, I., Tasma, I. M. and Hasanah, M. 1990. Effect of $\mathrm{N}$ and $\mathrm{P}$ fertilizers on sesamum growth yield and seed quality. Industrial Crop Research Journal. $3(1): 1-5$.

Deepika, C. and Anita, P. 2015. Effect of Zinc and Boron on Growth, Seed Yield and Quality of Radish (Raphanus sativus L.) cv. Arka Nishanth. Current Agriculture Research Journal. 3(1): 8589.

Dileepkumar, M. A., Vyakaranahal, B. S. and Deshpande, V. K. 2009. Influence of pelleting with micronutrients and botanical on growth, seed yield and quality of vegetable cowpea. Karnataka Journal of Agriculture Science. 22(4):898- 900.

El-Damarany, A. M., El-Shaikh, K. A. A., Obiadalla-Ali, H. A. and Abdel-Kader, M. M. 2016. Effect of Nitrogen and Potassium Fertilization on Seed Production of Onion (Allium cepa L.) Improved Giza 6 Cultivar. American-
Eurasian Journal Agriculture and Environment Science. 16(7): 12961303.

Geetharani, P., Manivannan, M. I., Ponnuswamy, A. S. 2008. Seed production of onion as influenced by the application of growth regulators and nutrients. Asian Journal of Horticulture. 3(2):301-303.

Hamsaveni, M. R., Kurdikeri, M. B., Shekhargouda, M., Shashidhara, S. D., Dharmatti, P. R. 2003. Effect of Gypsum and Boron on Seed Yield and Quality on Tomato Cv. Megha. Karnataka Journal of Agriculture Science. 16(3):457-459.

He, W. and Chen, D. 1996. Effect of different levels of fertilizer application on growth of tomato seedlings grown in seedling trays. Beijing Agriculture Science. 4(2):22-24.

Jadhao, B. J., Kulwal, L. V., Mahakal, K. G. 1999. Effect of nitrogen, phosphorus and potassium on growth and seed yield of radish (Raphanus sativus L.). Vegetable science. 26(1): 95-96.

Kabir, A., Ali, A., Waliullah, M. H. 2013. Effect of spacing and sowing time on growth and yield of carrot (Daucus carota L.). International Journal of Sustainable Agriculture. 5(1): 29-36.

Marks, S. J., Vyakarnahal, B. S., Shekhargouda, M., Patil, V. S., Patil, M. S. 2006. Effect of fertilizer and spacing levels on seed yield and its attributes in ajowain. Karnataka Journal of Agricultural Sciences. 19(1):124126.

Rahim, M. A., Hakim, M. A. and Rahman, M. M. 1997. Effect of nitrogen and potash on the yield of onion seed. Bangladesh Journal Science Indian Research. 32(2): $322-325$

Raina, J. N. 2002. Drip irrigation and fertigation: Prospects and Retrospect's in temperate fruit production: In: 
Enhancement of temperate fruit production in changing climate (Eds) $\mathrm{K}$. K. Jindal and D. R. Gautam. University of Horticulture and Forestry, Solan. pp. 296-301.

Raina, J. N., Sharma, T., Shashi, S. 2011. Effect of drip fertigation with different fertilizers on nutrient distribution in soil, leaf nutrient content and yield of apricot (Prunus armeniaca L.). Journal of the Indian Society of Soil Science. 59: 268-277.

Rajangam, J. 1991. Studies on the influence of planting density and nitrogen levels on growth, yield and quality of chilli (Capsicum annuит L.) cv. CO 2. M Sc Thesis, Tamil Nadu Agriculture University Coimbatore, Tamil Nadu, India.
Sharma, S. K. 1995. Response of boron and calcium nutrition on plant growth and seed yield and tomato. Vegetable Science. 22: 27-29.

Singh, K. 2003. Approaches for sustainable development of onion and garlic. National Horticulture Research and Development Foundation Report. 1-13.

Suthar, M. R., Singh, G. P., Rana M. K., Makhan, L. 2005. Growth and fruit yield of brinjal (Solanum melongena L.) as influenced by planting dates and fertility levels. Crop Research Journal. 30(1):77-79.

Verma, S. K., Singh, S. S. and Awasthi, C. P. 1995. Response of zinc and boron fertilization on yield and quality fruits at different stages of picking. Vegetable Science. 22: 5-8.

\section{How to cite this article:}

Himangini and Kanwar. H.S. 2020. Effect of Drip Fertigation and Boron Foliar Spray on Growth, Seed Yield and Quality of Onion (allium cepa 1.) Cv. Palam lohit. Int.J.Curr.Microbiol.App.Sci. 9(01): 1314-1321. doi: https://doi.org/10.20546/ijcmas.2020.901.145 